\title{
ФАКТОРЫ, ВЛИЯЮЩИЕ НА РЕЗУЛЬТАТЫ УПРАВЛЕНИЯ ПРОДАЖАМИ В УСЛОВИЯХ КОНКУРЕНЦИИ В СФЕРЕ УСЛУГ (НА ПРИМЕРЕ УСЛУГ РОЗНИЧНОЙ ТОРГОВЛИ)
}

\begin{abstract}
АНнотАЦия. Успешность управления продажами, в том числе и в розничной торговле, зависит от полноты учета влияющих факторов, как на этапе принятия управленческих решений, так и на этапе их реализации. Содержание влияющих факторов в значительной мере зависит от подхода $\kappa$ определению места управления продажами в системе управления организацией. В этой связи в статье проведено исследование узкого, расширенного и широкого подходов, в трактовке автора, к процессу управления продажами и показано что более обоснованным широким подходом является системный подход, в рамках которого предполагается, что результаты управления продажами зависят не только от таких факторов как применяемые технологии продаж, обоснованность планирования, организации, мотивации, контроля и регулирования процесса продажи, но, в первую очередь, от такого фактора как соответствие реализованной рыночной политики значимой комбинации ценностей искомых целевыми покупателями, определяющего выбор магазина целевыми покупателями, возможность совершения в нем необходимых объемов покупок за период, покупательскую лояльность. Для измерения обозначенного фактора предложен соответствующий интегральный показатель и алгоритм его расчета Применение этого показателя позволит повысить объективность оценки работы торгового персонала и проблем торгового предприятия в разрезе каждого направления рыночной политики. КЛЮчЕВЫЕ СЛОВА. Концепция управления продажами; рыночная политика; факторы управления продажами; интегральный показатель соответствия.

ИНФОРМАЦИЯ О СТАТЬЕ. Дата поступления 15 апреля 2017 г.; дата принятия к печати 11 мая 2017 г.; дата онлайн-размещения 19 июня 2017 г.
\end{abstract}

N. G. Novikova

Baikal State University, Irkutsk, Russian Federation

\section{FACTORS INFLUENCING THE RESULTS OF SALES MANAGEMENT IN TERMS OF COMPETITION IN SERVICE SPHERE (AS EXEMPLIFIED BY RETAIL TRADE SERVICES)}

\begin{abstract}
Successfulness in sales management, including retail trade, depends on fullness of considering the influential factors, both at the stage of making managerial decisions and at the stage of their implementation. The content of the influential factors considerably depends on the approach to determining the place of sales management in the organization's management system. In that context, the article carries out an investigation of narrow, expanded and wide approaches, in the author's interpretation, to the process of sales management and shows that the system approach is a more substantiated wide option, which framework assume that sales management results depend not only on such factors as existing sales technologies, feasibility of planning, organization, motivation, control and sales process regulation, but first and foremost on such a factor as correspondence of the implemented market policy to a significant combination of values sought by target buyers, that determines the choice of the shop by target buyers, possibility of shopping in necessary sales amount over a certain period, the buying loyalty. In order to measure the outlined factor, the article offers a corresponding integral indicator and the algorithm of its calculation. The use of this indicator will allow to increase the objectivity of assessing trade personnel's performance and the problems of the commercial enterprise in terms of each direction of the market policy.
\end{abstract}

(C) Н. Г. Новикова, 2017

\section{Baikal Research Journal}


KEYWORDS. Concept of sales management; market policy; factors of sales management; integral indicator of correspondence.

ARTICLE INFO. Received April 15, 2017; accepted May 11, 2017; available online June 19, 2017.

Конкурентоспособность любой коммерческой организации в условиях рынка при прочих равных условиях в значительной мере зависит от объема продаж и скорости оборота за период продукции и товаров. Нет необходимой скорости, объема выходящего потока при запланированных издержках — нет конкурентоспособности, а зачастую, нет и самой организации. Это определяет актуальность вопросов управления продажами, которым в последние годы уделяется много внимания как в теории, так и на практике, последнее подтверждается высокой популярностью бизнес-тренингов и семинаров по обозначенной теме. Очевидно, что успех любых управленческих усилий, в том числе и в сфере управления продажами, зависит от степени полноты учета влияющих на результаты факторов. Изучение специальной литературы [1-8], показало, что вопрос влияющих на результаты управления продажами факторов, исследован недостаточно. Проблема, в значительной мере, обусловлена тем, что несмотря на множество публикаций по вопросам управления продажами, отсутствует единство мнений авторов не только по отдельным аспектам, но и по самой концепции управления продажами, определяющей его место в системе управления организацией, от которого зависит видение, влияющих на результаты факторов. Очевидно, что такое положение в какой-то степени зависит от различий в условиях функционирования организаций и предприятий на В2В и В2С рынках. Однако при этом суть дела не меняется и обеспечение необходимых для выживания и успеха на рынке объемов продаж и скорости оборота, в том числе за счет более полного учета влияющих на них факторов, несомненно является важным направлением исследований.

Целью настоящей статьи является рассмотрение места управления продажами в системе рыночно-ориентированного управления розничной торговой организацией в условиях конкуренции и разработка подхода к оценке влияния на выполнение плана продаж такого фактора как адекватность рыночной политики.

Для достижения обозначенной цели в статье поставлены и решены следующие основные задачи:

1. Исследование концепций и подходов к управлению продажами продукции, товаров, представленных в специальной литературе.

2. Определение места управления продажами в системе рыночно-ориентированного управления торговой организацией и рассмотрение результатов реализации рыночной политики торговой организации как фактора, влияющего на результаты процесса управления продажами.

3. Разработка алгоритма расчета интегрального показателя оценки соответствия реализованной рыночной политики значимой комбинации ценностей, искомых целевыми покупателями торговой организации.

Исследование публикаций по вопросам о сущности управления продажами, позволило распределить их на три группы по такому признаку как широта подхода: узкий, расширенный и широкий.

При узком подходе управление продажами рассматривается в рамках стратегии ведения конкретной продажи и определяется той или иной ее технологией [6].

Сторонники подхода, который мы условно обозначили как расширенный, рассматривают управление продажами в контексте деятельности отдела продаж или иного аналогичного по реализуемым функциям подразделения организации. При этом рассматриваются известные функции управления: планирование; организация, в том числе найм, обучение торгового персонала технологиям продаж;

\section{Baikal Research Journal}

электронный научный журнал Байкальского государственного университета 
мотивация; контроль и регулирование [1]. Таким образом авторы рассматривают процесс управления продажами изолировано, как самостоятельный процесс. Очевидно в этом есть свой смысл, так как эта функциональная зона управления имеет свои специфические особенности, требующие определенных квалификационных знаний, умений и навыков в области организации и выполнения работы.

Если мы обратимся к специальной литературе посвященной розничной торговле, то увидим, что термин «управление продажами» самостоятельно применяется редко. Значительно более распространенным термином является «управление ассортиментом», где управление продажами занимает центральное место [9]. Рассмотрим место управления продажами в системе управления ассортиментом. Наши исследования процесса управления ассортиментом позволили выделить пять этапов. На рис. 1 представлена взаимосвязь между этапами управления ассортиментом, показан его итерационный характер.

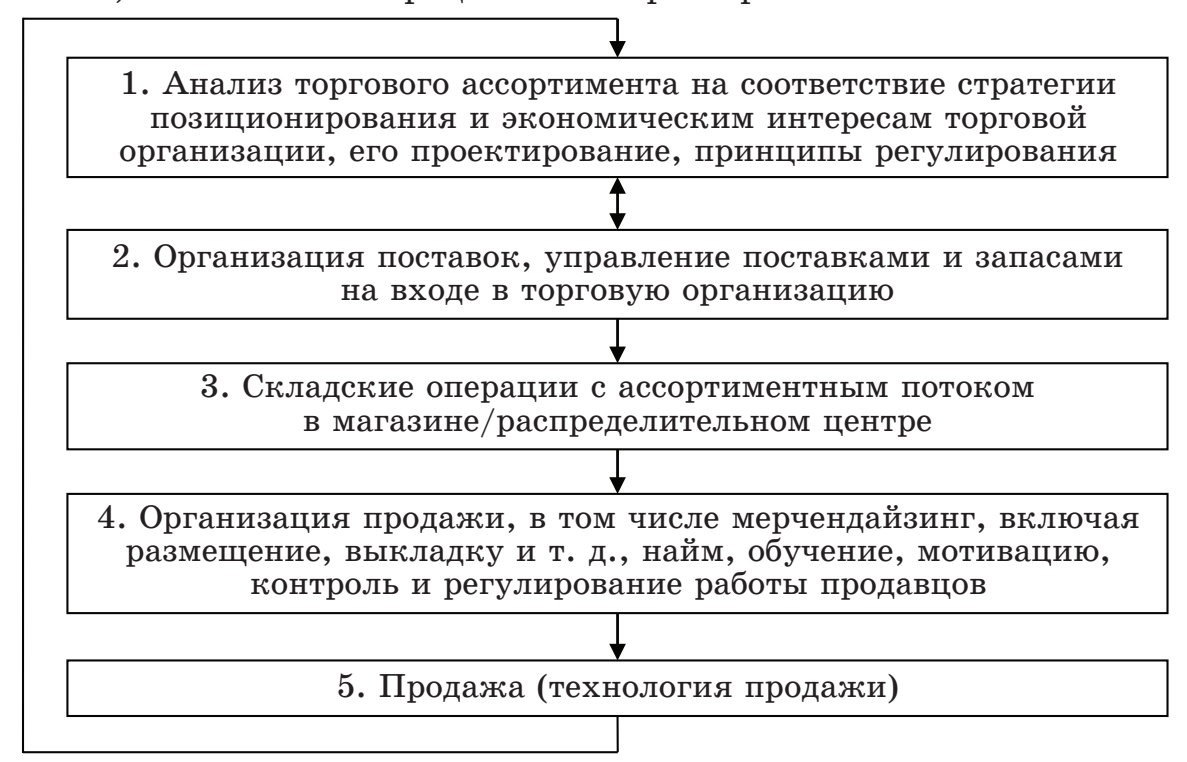

Рис. 1. Этапы управления ассортилентом в розничной торговле

Из рис. 1 становится ясно, что функции аналогичные деятельности отдела продаж выполняются на этапах 4 и 5 . При этом из рис. 1 видно, что в рамках каждого из предшествующих этапов действуют факторы, способные повлиять на результаты работы сотрудников непосредственно занимающихся организацией продажи и продажей. Например, срыв поставки, несоблюдение условий складирования и сроков отгрузки товаров со склада (распределительного центра торговой сети) окажут несомненное отрицательное влияние на товарооборот, несмотря на все усилия торгового персонала.

Однако рассмотрение управления продажами только в контексте технологии продаж, организации работы отдела продаж, управления ассортиментом (применительно к розничной торговле) является недостаточным. Ограниченность такого подхода проявляется в том, что в его рамках вся ответственность за выполнение разработанного плана продаж полностью возлагается на продавцов и их непосредственных руководителей. В действительности усилия этих сотрудников организации являются хотя и важными, но не единственными факторами выполнения плана продаж. Как видно из рис. 1 объем и скорость движения ассортиментного потока зависят также и от действий осуществляемых на этапах $1,2,3$. Но и это

\section{Baikal Research Journal}

электронный научный журнал Байкальского государственного университета 
еще не все. Выполнение плана продаж (товарооборота) в торговых организациях зависит не только от качества управления ассортиментом, но также и от других управленческих усилий, например, от управления ценой и др.

Сторонники широкого подхода к управлению продажами выходят за рамки отдела продаж и рассматривают этот процесс в контексте миссии, стратегий и, в частности, маркетинговой стратегии $[2 ; 3 ; 8]$.

Так в работах Ч. Фатрелла В. А. Вертоградова обозначено место стратегии продаж в деятельности компании. Авторы отмечают, что стратегия развития определяет стратегию маркетинга в которой закрепляется, что именно компания будет производить, кому, каким образом и по какой цене будет продавать. На ее основе вырабатывается стратегия продаж (стратегия сбыта), непосредственно определяющая, каким образом должна быть организована работа с клиентами компании, чтобы они стали покупателями [2;8].

Ч. Фатрелл трактует управление продажами как эффективное и результативное достижение целей, поставленных перед торговым персоналом с помощью планирования, подбора, обучения и руководства персоналом, а также контроля организационных ресурсов, он отмечает, что менеджеры по продажам отвечают за формирование сбыта, прибыли и удовлетворенность потребителей, соответствующих корпоративным целям [8, с. 25].

В работе Д. Джоббера и Дж. Ланкастера управление продажами рассматривается с позиции технологии продажи и организации продажи в том числе и в плане управления каналами распределения. Однако эти авторы посвящают свое внимание также и взаимосвязи маркетинга и продаж, рассматривают различные концепции маркетинга, противопоставляя концепцию маркетинга, нацеленную на продажи и концепцию маркетинга, нацеленную на потребителя. Они определяют место продаж в системе маркетинга-микс в его концепции $4 \mathrm{P}$, показывая, что продажи входят в такой его элемент как продвижение в виде персональных продаж [3, с. 35]. Ч. Фатрелл также показывает место продаж в системе маркетинга-микс, концепцию которого он трактует как 4P [8, с. 87].

Особенно интересным представляется подход к управлению продажами, изложенный в работе Р. Лукича. Он наряду с глубоким рассмотрением вопросов технологии продажи и ее организации в своей книге большое значение придает взаимосвязи отдела продаж с другими отделами, миссией компании в целом, ожиданиями потребителей: «Если в списке приоритетов вашей компании клиент стоит не на первом месте, значит, проблема в вашей компании, а не в клиенте! Действуете ли вы в одной команде с отделами маркетинга, производства, логистики, с разработчиками информационных систем и специалистами по их внедрению? Вокруг чего вы собираетесь сплотить команду продавцов? Есть ли у вас миссия? Стратегия? Ожидаете ли вы, что клиент будет верить в то, во что вы сами не верите? Говорите ли вы клиенту, что ваши конкуренты поставляют коробки с товаром, а вы - решения? Что вы имеете в виду? Верите ли в это сами?» [5, с. 15]. Интересен также подход этого автора, к так называемым, внутренним продажам. Суть внутренних продаж по мнению автора сводится к умению сотрудников отдела продаж «продать» собственной компании свой взгляд на организацию сбыта и свои подходы к продажам с целью заставить работать в интересах отдела другие подразделения компании и организовать эффективный сбыт ее продукции [Там же]. По нашем мнению, такой подход способен внести большой вклад в обеспечение межфункциональной и межиерархической интеграции решений и действий в организации, повысить уровень.

Все эти авторы рассматривают управление продажами преимущественно с позиции организаций-производителей продукции, и хотя они указывают на зависимость деятельности отдела продаж от целей и стратегий организации, но не рас-

\section{Baikal Research Journal}

электронный научный журнал Байкальского государственного университета 
сматривают их реализацию как фактор, влияющий на результаты деятельности отдела продаж и объективность оценки торгового персонала.

Однако системный подход как известно предполагает рассмотрение совокупности взаимосвязанных элементов как единого целого, объединенного общностью цели. При этом характер взаимосвязи таков, что воздействие на один из элементов системы приводит к изменению других ее элементов. Это подтверждается тем, что выполнение плана товарооборота, как результата управления продажами зависит не только от работы отдела продаж, категорийных менеджеров, продавцов, директоров и управляющих магазинами, но и от модели управления организацией в целом, а также от успешной реализации ее рыночной политики.

Под рыночной политикой розничной торговой сети понимается комплекс контролируемых коммерческих инструментов, посредством которых торговая организация, воздействуя на целевых покупателей, реализует свою стратегию позиционирования и конкурентную стратегию в целом.

Понятие «позиционирование» (стратегия позиционирования) неоднозначно трактуется в специальной литературе. Это определяет необходимость кратко рассмотреть существующие подходы и определить тот который будет использоваться в настоящей статье.

При узком, функциональном подходе к маркетингу, когда деятельность маркетинговой службы в организации рассматривается как функция вспомоществования сбыту и сводится к маркетинговым коммуникациям, позиционирование трактуется как некая оболочка, упаковка для товара, организации в целом, позволяющая отстроить их от предложений конкурентов и не связанная при этом с фактическим созданием ценностей, искомых целевыми потребителями, хотя и, зачастую, основывается на их знании.

Типичным высказыванием для сторонников функционального подхода к маркетингу является следующее: «Другими словами, позиционирование не затрагивает продукцию, а непосредственно направлено на мнение потребителя относительно данной продукции с помощью различных сообщений».

Постфункциональный подход к маркетингу определяет иную трактовку стратегии позиционирования (позиционирования), реализация которой затрагивает все направления рыночной политики и функциональные зоны управления организацией, непосредственно связывает его с реальным созданием значимой комбинации ценностей, искомых целевыми потребителями/покупателями. В соответствии с постфункциональным подходом к маркетингу и применительно к розничной торговле, позиционирование это разработка благоприятной позиции формата торговой сети, которую необходимо сформировать в сознании целевых покупателей на основании создания значимой комбинации искомых ими ценностей, с учетом позиций уже занятых конкурентами. По мнению М. Портера позиционирование составляет ядро конкурентной стратегии организации [10].

Рыночная политика является комплексом тактических решений, посредством осуществления которых реализуется стратегия позиционирования и в реальности создается значимая комбинация ценностей, искомых целевыми потребителями/ покупателями. Именно от этого при прочих равных условиях зависит придут ли целевые покупатели в наш магазин и совершат ли покупки в необходимом за период объеме.

Структура рыночной политики, зависит от видения руководителей организации. Ее концепция, определяющая направления/комплексы контролируемых коммерческих инструментов, при помощи которых организация осуществляет свои сделки и воздействует на рынок, формируется на основании методических подходов, по-разному называемых в специальной литературе. Наиболее извест-

\section{Baikal Research Journal}

электронный научный журнал Байкальского государственного университета 
ным их представлением являются различные концепции маркетинга-микс, объединяющие элементы, которые по своей сути и являются этими контролируемыми коммерческими инструментами. Однако они известны также и под другими названиями: активные переменные в мультиатрибутивной концепции магазина [11, с. 577], категории сделки в системе коммерческих операций [12, с. 11] и др.

Розничная торговля имеет свою существенную специфику деятельности, определяющие особенности ее рыночной политики. В связи с этим более обоснованной концепцией рыночной политики, по нашему мнению, является концепция, основанная на маркетинге-микс, включающем 7Р и рекомендуемой специалистами для сферы услуг. В адаптированном к торговле виде она может быть представлена следующими коммерческими инструментами торговой организации: товарный ассортимент, ценовой микс, местоположение (доступность), коммуникационный микс, персонал, процесс, физическая среда /физические свидетельства. При данной трактовке рыночной политики видно, что за управление продажами непосредственно отвечают два элемента: коммуникационный микс, включающий в себя личные продажи, его чаще всего связывают с технологией продажи и персонал, с этим элементом чаще всего связывают вопросы планирования, организации, мотивации, контроля и регулирования персонала. Для того чтобы рассмотреть влияние на продажи других элементов рыночной политики обратимся к рис. 2, на котором представлена итерационная модель рыночно-ориентированного управления торговой организацией, показывающая связь между стратегическими, тактическими и операционными решениями и действиями организации.

Как видно из рис. 2 достижение запланированных результатов по управлению продажами зависит не только от успешности организации продаж и совершенства применяемых технологий продажи, но также, и от того в какой мере остальные элементы рыночной политики нацелены на создание ценностей, искомых целевыми покупателями. От их правильной реализации зависит сама возможность продажи и потому степень их соответствия значимой комбинации ценностей, искомых целевыми потребителями следует рассматривать как один из влияющих факторов.

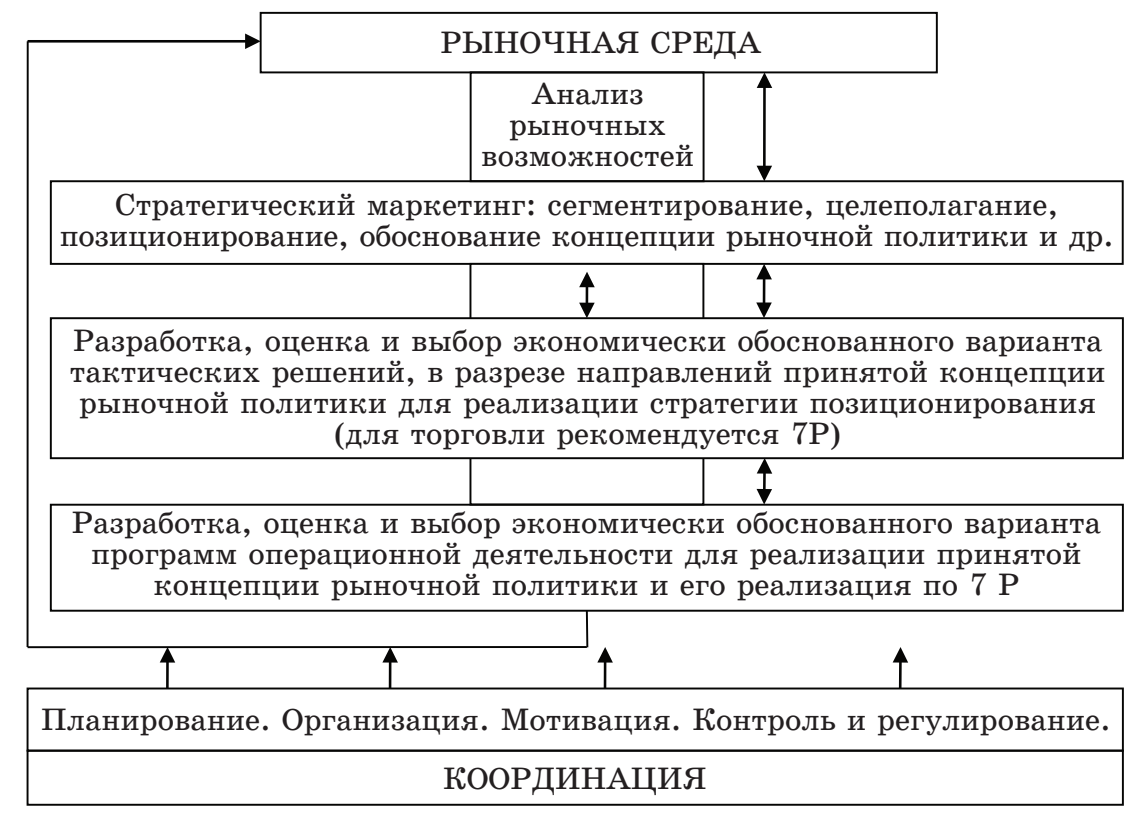

Рис. 2. Модель рыночно-ориентированного управления торговой организацией

\section{Baikal Research Journal}

электронный научный журнал Байкальского государственного университета 
Для измерения действия обозначенного фактора можно использовать показатель соответствия реализуемой рыночной политики значимой комбинации ценностей, искомых целевыми покупателями. Расчет этого показателя рекомендуется производить, применяя методы экспертных оценок, некоторые вопросы которых рассмотрены в [13]. Алгоритм расчета показателя представлен на рис. 3. Предложенный подход расчета интегрального показателя (рис. 3) предполагает, что он может изменяться от 0 до 1 . Чем ближе значение показателя к единице, тем адекватнее реализуемая рыночная политика значимой комбинации ценностей, искомых целевыми покупателями.

Шаг 1. Произвести экспертную оценку каждого элемента рыночной политики с позиции силы и направления его влияния (положительное/отрицательное) на значимую комбинацию ценностей, искомых целевыми покупателями, по любой балльной шкале, например по 5-ти балльной шкале: 5 - баллов максимальное положительное влияние, 0 - баллов отсутствие влияния (структура рыночной политики может быть представлена любой значимой для сектора бизнеса комбинацией контролируемых переменных, для розничной торговли в качестве основы рекомендуется концепция 7Р)

Шаг 2. Произвести экспертную оценку степени значимости вклада каждого элемента/направления рыночной политики в формирование значимой комбинации ценностей, искомых целевыми покупателями, распределив 100 баллов между всеми направлениями рыночной политики в зависимости о ощущаемой степени их важности в формировании значимой комбинации ценностей, искомых целевыми покупателями. Полученные баллы для каждого направления рыночной политики следует перевести в доли единицы, таким образом можно получить коэффициенты значимости направлений рыночной политики применительно к целевому рынку, изучаемого формата розничной торговли анализируемой сети. Их сумма равна 1.

Шаг 3. Произвести расчет интегральной оценки соответствия реализованной рыночной политики значимой комбинации ценностей, искомых целевыми покупателями, может быть получена как сумма произведений частных оценок и коэффициентов значимости для каждого направления рыночной политики по формуле:

$$
I=\frac{\sum_{i}^{n} O_{i} Z_{i}}{\sum_{i}^{n} O_{\max } Z_{i}}
$$

где $I$ - интегральный показатель соответствия рыночной политики значимой комбинации ценностей, искомых целевыми покупателями; $Z_{i}-$ коэффициент значимости $i$-го направления рыночной политики для данного формата торговли и его целевого рынка, $i$ - изменяется от $1, n, n-$ количество направлений рыночной политики, взятых для анализа торговой сети; $O_{i}$ - экспертная оценка соответствия $i$-го направления рыночной политики значимой комбинации ценностей, искомых целевыми покупателями анализируемого формата торговли; $O_{\max }$ - максимальная положительная по принятой шкале оценка соответствия $i$-го направления рыночной политики значимой комбинации ценностей, искомых целевыми покупателями анализируемого формата торговли

Рис. 3. Алгоритл расчета интегрального показателя соответствия рыночной политики значилой колбинации иенностей, исколых иелевыли покупателям

При этом особенное внимание следует уделять результатам экспертных оценок, полученных в рамках шага 1 и шага 2, поскольку они позволяют установить в рамках какого из направлений рыночной политики существуют наибольшие проблемы и какова значимость этого направления с точки зрения влияния на

\section{Baikal Research Journal}


привлечение целевых покупателей в магазины торговой сети. Применение интегрального показателя позволит получить следующие положительные результаты управления продажами и организацией в целом:

- повысится обоснованность, справедливость оценки работы торгового персонала, будут исключены ситуации, когда ответственность за невыполнение плана продаж (товарооборота) полностью возлагается на торговый персонал, это будет способствовать повышению его мотивации;

- повысится объективность оценки работы всех подразделений и проблем торговой организации в вопросах обеспечения ее рыночной ориентированности.

\section{Список использованной литературы}

1. Анучин А. А. Системный подход к управлению продажами / А. А. Анучин // Управление продажами. - 2016. - № 3. - С. 170-179.

2. Вертоградов В. А. Управление продажами / В. А. Вертоградов. - 2-е изд. - СПб. : Питер, 2011. - 236 с.

3. Джоббер Д. Продажи и управление продажами : учеб. пособие / Д. Джоббер, Дж. Ланкастер. - М. : Юнити-Дана, 2002. - 622 с.

4. Кент Т. Розничная торговля : учебник / Т. Кент, О. Омар. - М. : Юнити-Дана, 2015. -756 c.

5. Лукич Р. М. Управление продажами / Р. М. Лукич. - М. : Альпина Паблишер, 2013. -212 c.

6. Рекхэм Н. СПИН-продажи / Н. Рекхэм. - 7-е изд. - М. : Манн, Иванов и Фербер, 2014. $-336 \mathrm{c}$.

7. Спиро Р. Л. Управление продажами / Р. Л. Спиро, У. Дж. Стэнтон, Г. А. Рич. М. : Изд. дом Гребенникова, 2004. - 704 с.

8. Фатрелл Ч. Управление продажами / Ч. Фатрелл. - СПб. : Нева, 2004. - 640 c.

9. Бузукова Е. А. Закупки и поставщики. Курс управления ассортиментом в рознице / Е. А. Бузукова ; под ред. С. В. Сысоевой. - 2-е изд. - СПб. : Питер, 2015. - 416 с.

10. Портер М. Э. Конкуренция, обновленное и расширенное издание / М. Э. Портер. М. : Вильямс, 2012. -592 с.

11. Ламбен Ж. Ж. Менеджмент, ориентированный на рынок. Стратегический и операционный маркетинг / Ж.-Ж. Ламбен. - СПб. : Питер, 2005. - 800 с.

12. Кроуфорд $\Phi$. Миф о совершенстве: Почему великие компании не пытаются достичь совершенства во всем / Ф. Кроуфорд, Р. Мэтьюс. - М. : Росмэн-Пресс, 2005. - 239 с.

13. Новикова Н. Г. Управление конкурентоспособностью коммерческой организации: маркетинговый подход : учеб. пособие / Н. Г. Новикова. - Иркутск : Изд-во БГУэП, 2012. $-178 \mathrm{c}$.

\section{References}

1. Anuchin A. A. System approach to sales management. Upravlenie prodazhami $=$ Sales Management, 2016, no. 3, pp. 170-179. (In Russian).

2. Vertogradov V. A. Upravlenie prodazhami [Sales Management]. $2^{\text {nd }}$ ed. Saint Petersburg, Piter Publ., 2011. 236 p.

3. Jobber David, Lancaster Geoffrey. Selling \& Sales Management. Pearson Education Ltd., 1990. (Russ. ed.: Jobber D., Lancaster G. Prodazhi i upravlenie prodazhami. Moscow, Yuniti-Dana Publ., 2002. 622 p.).

4. Kent Tony, Omar Ogenyi Ejye. Retailing. London, Palgrave Macmillan, 2002. 544 p. (Russ. ed.: Kent T., Omar O. E. Roznichnaya torgovlya. Moscow, Yuniti-Dana Publ., 2015. 756 p.).

5. Lukich R. M. Upravlenie prodazhami [Sales Management]. Moscow, Al'pina Pablisher, 2013. $-212 \mathrm{~s}$.

6. Rackham Neil. SPIN-Selling (Russ. ed.: Rackham N. SPIN-prodazhi. $7^{\text {th }}$ ed. Moscow, Mann, Ivanov and Ferber Publ., 2014. 336 p.).

7. Spiro Rosann L., Stanton William J., Rich Gregory A. Management of a sales force. New York, McGraw-Hill, 2003. 672 p. (Russ. ed.: Spiro R. L., Stenton U. J., Rich G. A. Upravlenie prodazhami [Sales Management]. Moscow, Grebennikov Publ., 2004. 704 p.).

\section{Baikal Research Journal}


8. Futrell Charles M. Sales Management Fort Worth. Harcourt College Publ., 2001. 609 p. (Russ. ed.: Futrell Ch. Upravlenie prodazhami. Saint Petersburg, Neva Publ., 2004. 640 p.).

9. Buzukova E. A.; Sysoyeva S. V. (ed.). Zakupki i postavshchiki. Kurs upravleniya assortimentom $v$ roznitse [Purchasing and Suppliers. A Course of Assortment Management in Retail Trade]. Saint Petersburg, Piter Publ., 2015. 416 p.

10. Porter M. E. On Competition. Updated \& Expanded Edition. Harvard Business Review Press, 2008. 576 p. (Russ. ed.: Porter M. Konkurentsiya. Moscow, Viliyams Publ., 2012. 592 p.).

11. Lambin Jean-Jacques. Market-Driven Management: Strategic and Operational Marketing. New York, Palgrave Macmillan, 2000. 496 p. (Russ. ed.: Lambin J.-J. Menedzhment, orientirovannyi na rynok. Strategicheskii $i$ operatsionnyi marketing. Saint Petersburg, Piter Publ., 2005. 800 p.).

12. Crawford Fred, Mathews Ryan. The Myth of Excellence: Why Great Companies Never Try to Be the Best at Everything. New York, Crown Business, 2001. 230 p. (Russ. ed.: Crawford F., Mathews R. Mif o sovershenstve: Pochemu velikie kompanii ne pytayutsya dostich' sovershenstva vo vsem. Moscow, Rosmen-Press, 2005. 239 p.).

13. Novikova N. G. Upravlenie konkurentosposobnostyu kommercheskoi organizatsii: marketingovyi podkhod [Managing the Competitiveness of the Commercial Organization: a Marketing Approach]. Irkutsk, Baikal State University of Economics and Law Publ., 2012. 178 p.

\section{Информация об авторе}

Новикова Надежда Григорьевна - доктор экономических наук, профессор, кафедра логистики и коммерции, Байкальский государственный университет, 664003, г. Иркутск, ул. Ленина, 11; e-mail: ngn777@mail.ru.

\section{Author}

Nadezhda G. Novikova - Doctor habil. (Economics), Professor, Chair of Logistics and Commerce, Baikal State University, 11 Lenin St., 664003, Irkutsk, Russian Federation; e-mail: ngn777@mail.ru.

\section{Библиографическое описание статьи}

Новикова Н. Г. Факторы, влияющие на результаты управления продажами в условиях конкуренции в сфере услуг (на примере услуг розничной торговли) / Н. Г. Новикова // Baikal Research Journal. — 2017. — T. 8, № 2. — DOI: 10.17150/2411-6262.2017.8(2).19.

\section{Reference to article}

Novikova N. G. Factors influencing the results of sales management in terms of competition in service sphere (as exemplified by retail trade services). Baikal Research Journal, 2017, vol. 8, no. 2. DOI: 10.17150/2411-6262.2017.8(2).19. (In Russian).

\section{Baikal Research Journal}

